\title{
DECIMALS AND LIQUIDITY: A STUDY OF THE NYSE
}

\author{
Sugato Chakravarty \\ Purdue University \\ Robert A. Wood \\ University of Memphis \\ Robert A. Van Ness \\ University of Mississippi
}

\begin{abstract}
Using a carefully constructed matched sample of control (nondecimal) stocks, we isolate the effects of decimalization for a sample of NYSE-listed common stocks trading in decimals. We find that the quoted depth as well as the quoted and effective bid-ask spreads declined significantly following decimalization. Additionally, both the number of trades and trading volume declined significantly. Stock return volatilities display an initial increase but a decline over the longer term, probably as traders become more comfortable in their new milieu.
\end{abstract}

JEL Classifications: G14, G18

The theory is straightforward: As prices are quoted in smaller and smaller increments, there are more opportunities and less costs for dealers and investors to improve the bid or offer on a security. As more competitive bidding ensues, naturally the spread becomes smaller. And this means better, more efficient prices for investors.

\section{Arthur Levitt, Chairman SEC}

March 2000

\section{Introduction}

Between August 28, 2000, and January 29, 2001, the New York Stock Exchange (NYSE), through a series of incremental steps, began trading and quoting

Earlier drafts of this manuscript were entitled "Decimal Trading and Market Impact." We thank Robert Battalio, Tarun Chordia, Bill Christie, Mike Cooper, Amy Edwards, Gene Finn, Michael Goldstein, Robert Jennings, Ananth Madhavan, Tim McCormick, William T. Moore (the editor), Eric Sirri, George Sofianos, Bonnie Van Ness, Richard Warr, and Robert Wood Jr. for comments on earlier drafts, and to the seminar participants in the 14th annual Financial Markets Research Center conference at Vanderbilt and, especially, Ingrid Werner for her comments. Wonil Hwang and Dan Zhou provided research assistance in an earlier draft and Jang-Chul Kim provided exceptional research assistance during the revision of this paper. Finally, we thank Stephen P. Harris of Bridge Information Systems who provided some of the data used in the initial stages of the project. 
all its listed securities in increments of 1 cent. ${ }^{1}$ Decimalization has finally arrived in Wall Street! A 200-year tradition of trading in fractions is history. We use this act of decimalization on the NYSE as a natural experiment to determine its effect on variables related to market liquidity.

According to the NYSE, the reduction in minimum price increment to sixteenths, in June 1997, was an interim step in a move toward the decimalization of prices and price increments (Jones and Lipson 2001). Although it is tempting to think of decimalization as merely a continuation in the process of tick-size reduction, it is actually much more than that. Although the move to sixteenths resulted in a doubling of the price points, or ticks, the move to decimals (from sixteenths) resulted in a six-fold increase in ticks. This increase in price points also decreases the costs for traders to jump in front of other orders (with decimal prices, traders can jump a pricing grid for as little as 1 cent per share, as compared to about 6.25 cents a share under sixteenths).

The NYSE's board approved conversion to decimal pricing in June 1997 to make prices more easily comprehensible by investors, reduce spreads, and bring the United States into conformity with international practices. Market liquidity is of paramount importance to both suppliers and demanders of capital as well as to regulators entrusted with the mandate of maintaining a fair and orderly market. Although there is little debate that prices are easily understood under the decimal system, whether this has actually influenced market liquidity is an empirical issue and is a focus of the current study.

Previous studies, both theoretical and empirical, fall into a large body of research on tick-size reductions. For examples of theoretical research, see Chordia and Subrahmanyam (1995) and Harris (1994); for examples of empirical research, see Goldstein and Kavajecz (2000) and Jones and Lipson (2001). Bacidore, Battalio, and Jennings (2001a, 2001b) examine order-submission strategies, liquidity, and execution quality around the NYSE's change to decimals. Bessembinder (2002) and Chung, Van Ness, and Van Ness (2002) examine matched sets of NYSE and NASDAQ stocks. These authors find that the NYSE has lower spreads than NASDAQ when equally weighted measures of spread are examined, but the NYSE and NASDAQ have generally similar spreads when volume weighted metrics are used.

Our study differs from previous studies in that we examine only the NYSE. Moreover, because the NYSE converted to decimal pricing via a phased pilot program, we can form a matched, nondecimal control sample. We use the matched securities to control for market effects that might occur coincidentally with the

\footnotetext{
${ }^{1}$ Specifically, the NYSE lowered the minimum tick size to a penny for seven securities on August 28, 2000, 57 more securities on September 25, 2000, and an additional 94 securities on December 5, 2000. All remaining securities began trading in decimals on January 29, 2001.
} 
decimalization of the pilot securities' prices. In addition, we use the decimal-pilot securities as a control sample for the matched (nondecimal) securities when trading in the matched securities was decimalized on January 29, 2001. We examine the behavior of both groups of stocks up to March 30, 2001. Our final decimal sample of 79 common stocks (of 158 total securities included in the decimal pilot) is derived after filtering all noncommon stocks (preferred stocks, closed-end funds, American Depositary Receipts [ADR], etc.) and ensuring certain minimum price and trading conditions are satisfied. These filters are incorporated to minimize confounding effects and are detailed later. The 79 common stocks from the decimal pilot are denoted decimal stocks, and the 79 corresponding (nondecimal) stocks created by matching are labeled control stocks. We then use tick-by-tick data for these stocks to investigate the effect of decimal trading on the various facets of market liquidity.

Our investigation period comprises three periods surrounding the decimal pilot in the NYSE: (1) a pre-decimal period (before any NYSE stock was trading in decimals), (2) a decimal trial period (when only securities in the NYSE's decimal pilot were trading in decimals), and (3) an all-decimal period (when all NYSE stocks were trading in decimals). Including the last period enables us to study the long-term effects of decimalization on the stocks in our sample.

We find that both bid and ask quote increments of 5 cents or less appear to be used actively by the market, and that quoted spreads, effective spreads, and depths declined significantly following decimalization. We find that both trades and trading volume declined significantly in all trade size categories as well as in all stock size categories. Stock return volatilities display an initial increase then a decline over the longer term, probably as traders become more comfortable in their new milieu. Although there is some evidence to suggest increased activity among the regional stock exchanges with regard to bid-ask quote adjustment frequencies, autoquotes (a bid-ask quote generated automatically by computers in regional exchanges positioned just outside the prevailing highest price to buy and the lowest price to sell), and best bid and offer (BBO) times, in the wake of decimalization, the evidence is not strong and the NYSE appears to remain in the lead in all these categories.

Our results have important policy implications as the debate continues over the benefits, costs, and effects of decimalization. The fact that quote increments of 5 cents or less appear to be used actively following decimalization may imply that minimum prices increments of one-eighth or one-sixteenth may have represented barriers to price competition. Furthermore, the proponents of decimalization argue that decimals allow for more efficient price discovery without adversely affecting liquidity. Opponents claim that decimals will result in less liquid, high-volatility markets. Our results, however, provide a mixed verdict on the issue of liquidity and volatility. At the very least, information about the available supply and demand schedule outside the BBO must be made available to market participants. The NYSE 
has already started publicly providing information on the depth of the limit order book. $^{2}$

\section{Data and Test Method}

\section{Sample Stocks}

The stocks in the decimal pilot were chosen based on several criteria developed by the NYSE, along with a securities-industry committee. These criteria include stocks that have varying levels of daily trading activity, that trade on multiple exchanges, that are part of an index, that are underlying issues for multiplelisted options; and that may have corporate action pending. To avoid introducing confounding effects, we eliminate all preferred and convertible preferred stocks, closed-end funds, ADRs, stocks with abnormally low average daily trading volume, and stocks with no trading in at least one day over the study period. We also exclude all common stocks below $\$ 5$ and above $\$ 150$ and stocks in the decimal pilot that moved from NASDAQ to NYSE during the study period. Our final sample comprises 79 NYSE-listed common stocks selected for trading in decimals under the three phases of the decimal pilot.

\section{Selection of Control Stocks}

Investigating the effect of decimalization on the pilot stocks after the event is valid under the assumption that the market has no underlying changes over the entire examination period. In the presence of market trends, however, it is impossible to tell whether an effect is due to decimalization or to market trends. We isolate the effect of decimalization, independent of market trends, by examining a matching sample of stocks identical to the control stocks in every way except that they do not trade in decimals. Accordingly, the control stocks are selected from the NYSE stocks with the same industry codes and security types (e.g., common stock) as the particular decimal stock. The control stock is now chosen to minimize the expression,

$$
\sum_{k}\left(c_{j}^{k}-c_{i}^{k}\right)^{2} /\left[\left(c_{j}^{k}+c_{i}^{k}\right) / 2\right]
$$

where $c_{i}^{k}\left(c_{j}^{k}\right)$ is the decimal (matched) stock measurement of characteristic $k$. Characteristics controlled include stock price, equity market capitalization, volatility, and trading volume. In addition to minimizing the sum, no single element of the

\footnotetext{
${ }^{2}$ Specifically, the NYSE, beginning March 19, 2001, started disseminating depth indications on eight of its stocks. Its purpose is to show investors that there is a meaningful number of shares of a given stock available beyond the best price being bid and offered for the stock (Wall Street Journal, March 15, 2001, C1). This has now been expanded to all NYSE-listed securities.
} 
summation is allowed to exceed unity. This approach is used by Chung, Van Ness, and Van Ness (2002) in selecting matching (control) samples. It should be noted, however, that we go further by employing an optimization model that simultaneously considers all possible decimal-control pairs and selecting only those pairs that minimize the overall distances (see McInish and Wood 1986).

We obtain tick-by-tick transaction and quote data for each stock in our decimal and control stock sample over the three periods of study: (1) October 2, 2000, to January 26, 2001 (the decimal trial period, when only some chosen stocks in the NYSE were trading in decimals, with the remaining stocks trading in sixteenths), ${ }^{3}$ (2) January 29, 2001, to March 30, 2001 (the all-decimal period, when all NYSE stocks were trading in decimals), and (3) April 1, 2000, to June 30, 2000 (the pre-decimal period, when no NYSE stocks were trading in decimals but, rather, were trading in sixteenths).

\section{Decimalization and Bid and Ask Quotes}

The quote data are error filtered, and all quotes with missing values, negative and zero spreads, and quoted spreads greater than $\$ 2$ are eliminated. This removes less than $1 \%$ of the quotes. ${ }^{4}$ The corresponding transactions prices are also examined (and filtered) for potential errors. We begin our investigation by providing in the following section metrics related to bid-ask quotes based on the NYSE-listed decimal and control stocks in our sample over the three periods of study.

\section{BBO Quotes and Quote Change Characteristics}

There exists, at any time, a set of bid and ask quotes that represents the highest price to buy and the lowest price to sell. In a continuous auction market such as the NYSE, a specialist posts quotes in stocks composed of a combination of own interest and the interest of the public limit orders competing directly with

\footnotetext{
${ }^{3}$ We choose the start date of the decimal trial period as October 2 - that is, week 6 onward, relative to the start of phase I decimal pilot in August 28, 2000 - to provide enough time for market participants to have found an equilibrium trading pattern because private communications with professional traders confirm that significant learning took place in the first few weeks of commencement of the decimal pilot as traders freely experimented with the new system.

${ }^{4}$ The comparison of trades and quotes also requires that data from Consolidated Tape Association (CTA) and CQS be merged by time. For various technical reasons (see Blume and Goldstein 1997), not Q2 only can the time stamps be in error but also the sequencing of trades in the same stock can be wrong. We considered using the Blume and Goldstein (1997) formula to adjust our quote time stamps the same trading day, but the quote adjustment algorithm Blume and Goldstein develop is based on 10-year-old data contained in the NYSE Trades, Orders, Reports, and Quotes (TORQ) database. Extensive improvements in trade recording systems have been implemented in the intervening years and there is currently a sense that adjusting quote time stamps is no longer appropriate.
} 
the specialist (for an analysis of quotes from the limit order book and specialist see Chung, Van Ness, and Van Ness 1999). Such competition can also come from the regional stock exchanges posting quotes simultaneously in those stocks. In short, BBOs are generated by the specialist and public limit orders originating both in the primary market and in the regional stock exchanges, which dictates which exchange at any time has the BBO quotes. The fraction of time that any exchange has the BBO is an indication of its dominance in terms of liquidity supply and price discovery.

We begin by investigating the fraction of total trading time that a BBO is in effect across the regional exchanges for decimal and control stocks over all three periods of interest. We find that NYSE dominates the BBO times in both decimal and control stocks over all three periods. ${ }^{5}$

Next, we investigate the frequency of changes in the BBOs. The purpose is to see whether all penny increments are used after decimalization or if the BBO quote changes are loading up only on certain penny increments. Specifically, we sum the number of times the best prevailing bid (ask) changes by 1 cent, 2 cents, and so on, for all the decimal and control stocks in our sample and over the decimal trial and all-decimal periods.

Over the decimal trial period, we find that about $25 \%$ (40\%) of quote changes in control (decimal) stocks occur at 6 cents or less (one-sixteenth) and $52 \%(76 \%)$ at 12 cents or less (one-eighth), and only about $6 \%$ of the changes in decimal stocks occur at 1 cent. Over the all-decimal period, 36\% (34\%) of quote changes in control (decimal) stocks occur at 6 cents or less and $70 \%(68 \%)$ at 12 cents or less, and only about $5 \%$ of the changes in both decimal stock and control stocks occur at 1 cent. Thus, although quote increments of less than 6 cents appear to be used actively by the market in decimal stocks, the evidence is by no means overwhelming. Also, the relative frequency of quote increments of 6 cents or less appears to have diminished significantly.

Given the reasonable use of increments of 5 cents or less in the BBOs noted earlier, a related question is which regional exchanges are responsible for this narrowing of the BBOs. Specifically, we examine 1 cent to 5 cent quote changes (at the best bid and at the best ask) classified by the regional exchanges quoting them over both decimal trial and all-decimal periods. We find that the NYSE has by far the greatest number of 1 cent to 5 cent changes, and the NASDAQ is competitive in all 5 cent categories. Overall, it appears that even though the regional exchanges are actively contending for quote changes, the NYSE is still leading the way.

Various researchers examine the issue of dynamic quote behavior both in the context of increased fragmentation of U.S. equity orders and in the context of price discovery (e.g., see Hasbrouck 1995). The NYSE and the regional exchanges

\footnotetext{
${ }^{5}$ This finding is consistent with the findings of Blume and Goldstein (1997) during one-eighth tick sizes and Bessembinder (2001) during one-sixteenth tick sizes.
} 
are electronically linked and all trades and quotes are disseminated by a central transmission authority (the CTA). Although the regional exchanges sometimes establish the BBO, frequently they choose to extract themselves from active quote competition in listed stocks by programming a computer to intercept all NYSE and American Stock Exchange (AMEX) quotes and immediately generate a new quote of their own by adding a delta to the ask and subtracting a delta from the bid, with 100 shares bid and 100 shares offered (the bid and ask depths). The mechanism(s) by which the regional quotes default to the BBOs, widened by a small arbitrary amount, are known as autoquotes (see Chordia, Roll, and Subrahmanyam [2001] for a discussion of autoquotes). Thus, an autoquote is effectively a nonquote whereby regional specialists signal that they are willing to supply liquidity but not at the best prices. Trades occur on the regional exchanges during periods of autoquoting, but those trades must match (or improve) the existing BBO. Our algorithm identifies an autoquote as any regional or third-market quote that brackets the existing NYSE or AMEX quote.

With the advent of decimalization it is reasonable to anticipate a change in the pattern of posting quotes off the BBO by liquidity providers wishing to earn more than a 1 cent to 2 cent spread. With the relatively thin book that naturally results from tighter spreads, significant buy-sell programs could easily march up or down the book and hit limit orders away from the minimum tick size.

We examine the percentage of time the NYSE-listed decimal and control stocks are autoquoted over the three periods of interest in the various regional exchanges. Specifically, we compute a simple percentage of all autoquotes from a given regional over all quotes from the regionals and the NYSE. Although tests reveal (results not reported) that the percentage of autoquoting is statistically similar for decimal and control stocks in the pre-decimal period, autoquoting increased significantly over the decimal trial period in decimal stocks relative to control stocks in most regional exchanges. Specifically, Cincinnati, Pacific, and Chicago, among the regionals, appear to have the highest increases of autoquoting in decimal stocks. This increase in autoquoting is perhaps an indication of the uncertainty felt by these regionals in their role as liquidity suppliers in a post-decimal world.

\section{Quoted and Effective Bid-Ask Spreads}

A traditional measure of market liquidity is the quoted bid-ask spread capturing the ex ante transactions cost. Researchers argue for a related measure, the effective spread, ${ }^{6}$ which measures the ex post transaction cost, contending that the quoted bid-ask spread is no longer an accurate measure of transaction costs when trades are executed inside the prevailing quoted spread.

${ }^{6}$ Effective spread is formally defined as twice the absolute difference between the transaction price and the midpoint of the prevailing BBO prices. 
TABLE 1a. Distribution of Odd and Even Tick Bid-Ask Spreads Among Regional Exchanges.

\begin{tabular}{|c|c|c|c|c|c|}
\hline \multirow[b]{2}{*}{ Exchanges } & \multirow[b]{2}{*}{ Ticks } & \multicolumn{2}{|c|}{$\begin{array}{c}\text { Decimal Trial Period } \\
\text { (October 2, 2000-January 26, 2001) }\end{array}$} & \multicolumn{2}{|c|}{$\begin{array}{c}\text { All-Decimal Period } \\
\text { (January 29, 2001-March 30, 2001) }\end{array}$} \\
\hline & & $\begin{array}{l}\text { Decimal Stocks } \\
\quad \text { Cum } \%\end{array}$ & $\begin{array}{l}\text { Control Stocks } \\
\quad \text { Cum \% }\end{array}$ & $\begin{array}{l}\text { Decimal Stocks } \\
\quad \text { Cum \% }\end{array}$ & $\begin{array}{l}\text { Control Stocks } \\
\quad \text { Cum \% }\end{array}$ \\
\hline \multirow[t]{2}{*}{ Boston } & Even ticks & $52.69 \%$ & $100.00 \%$ & $51.45 \%$ & $51.43 \%$ \\
\hline & Odd ticks & $100.00 \%$ & $100.00 \%$ & $100.00 \%$ & $100.00 \%$ \\
\hline \multirow[t]{2}{*}{ Cincinnati } & Even ticks & $54.12 \%$ & $100.00 \%$ & $52.26 \%$ & $53.45 \%$ \\
\hline & Odd ticks & $100.00 \%$ & $100.00 \%$ & $100.00 \%$ & $100.00 \%$ \\
\hline \multirow[t]{2}{*}{ Chicago } & Even ticks & $52.78 \%$ & $99.97 \%$ & $52.31 \%$ & $52.51 \%$ \\
\hline & Odd ticks & $100.00 \%$ & $100.00 \%$ & $100.00 \%$ & $100.00 \%$ \\
\hline \multirow[t]{2}{*}{ NYSE } & Even ticks & $46.33 \%$ & $99.99 \%$ & $45.28 \%$ & $44.27 \%$ \\
\hline & Odd ticks & $100.00 \%$ & $100.00 \%$ & $100.00 \%$ & $100.00 \%$ \\
\hline \multirow[t]{2}{*}{ Pacific } & Even ticks & $50.52 \%$ & $100.00 \%$ & $52.63 \%$ & $50.87 \%$ \\
\hline & Odd ticks & $100.00 \%$ & $100.00 \%$ & $100.00 \%$ & $100.00 \%$ \\
\hline \multirow[t]{2}{*}{ NASDAQ } & Even ticks & $51.44 \%$ & $99.97 \%$ & $60.54 \%$ & $59.91 \%$ \\
\hline & Odd ticks & $100.00 \%$ & $100.00 \%$ & $100.00 \%$ & $100.00 \%$ \\
\hline \multirow[t]{2}{*}{ Philadelphia } & Even ticks & $52.75 \%$ & $100.00 \%$ & $51.07 \%$ & $51.07 \%$ \\
\hline & Odd ticks & $100.00 \%$ & $100.00 \%$ & $100.00 \%$ & $100.00 \%$ \\
\hline
\end{tabular}

Note: The sample comprises selected NYSE-listed common stocks included in the decimal pilot and their corresponding NYSE-listed matched control stocks over the decimal trial and all-decimal periods. The cumulative percentages (Cum \%) presented here are computed from all (not just the best bid and offer) bid and ask quotes originating from the various exchanges. Only quoted spreads of up to $\$ 1$ are considered for this table. More than $95 \%$ of all quoted spreads are within $\$ 1$.

We begin our investigation into spreads by examining in Table 1a the distribution of all quoted spreads originating from the national and regional exchanges in terms of odd and even ticks for decimal and control stocks, over both the decimal trial and all-decimal periods. Over the decimal trial period, the decimal stocks are almost evenly split between even and odd ticks in every exchange, whereas the control stock spreads over the same period appear to be overwhelmingly on even ticks. Recalling that the control stocks over this period were trading in sixteenths, the implication is that the smallest possible spread in those stocks was $\$ 2 / 16$ or $\$ 1 / 8$. Over the all-decimal period when all stocks are trading in decimals, the quotes for both the decimal and control stocks originating on the regionals are almost evenly divided into even and odd ticks. The exceptions are the NYSE (NASDAQ), which shows significantly more odd (even) ticks in all stocks. Thus, about 45\% (61\%) of all ticks from the NYSE (NASDAQ) appear to be at even ticks.

Table $1 \mathrm{~b}$ refines the examination of quoted spreads by investigating the distribution of only the BBO spreads (in cents) for decimal and control stocks over the decimal trial and all-decimal periods. Decimal stocks display a higher frequency of spreads at 6 cents or less relative to the control stocks $(51 \%$ vs. $42 \%)$ over the decimal trial period. Decimal stocks continue to display greater dispersion even at spreads of 25 cents (or less). In examining the performance of decimal stocks over 
TABLE 1b. Distribution of Best Bid and Offer (BBO) Quoted Spreads of NYSE-Listed Decimal and Control Stocks.

\begin{tabular}{|c|c|c|c|c|}
\hline \multirow[b]{2}{*}{$\begin{array}{l}\text { Quoted } \\
\text { Spread Cents }\end{array}$} & \multicolumn{2}{|c|}{$\begin{array}{c}\text { Decimal Trial Period } \\
\text { (October 2, 2000-January 26, 2001) }\end{array}$} & \multicolumn{2}{|c|}{$\begin{array}{c}\text { All-Decimal Period } \\
\text { (January 29, 2001-March 30, 2001) }\end{array}$} \\
\hline & $\begin{array}{c}\text { Decimal Stocks } \\
\text { Cum } \%\end{array}$ & $\begin{array}{c}\text { Control Stocks } \\
\text { Cum\% }\end{array}$ & $\begin{array}{l}\text { Decimal Stocks } \\
\text { Cum\% }\end{array}$ & $\begin{array}{c}\text { Control Stocks } \\
\text { Cum } \%\end{array}$ \\
\hline 1 & $11.86 \%$ & $0.00 \%$ & $10.50 \%$ & $10.97 \%$ \\
\hline 2 & $21.81 \%$ & $0.00 \%$ & $19.72 \%$ & $21.37 \%$ \\
\hline 3 & $29.46 \%$ & $0.00 \%$ & $27.29 \%$ & $29.63 \%$ \\
\hline 4 & $36.58 \%$ & $0.00 \%$ & $34.59 \%$ & $37.38 \%$ \\
\hline 5 & $44.89 \%$ & $0.00 \%$ & $42.73 \%$ & $45.48 \%$ \\
\hline 6 & $51.26 \%$ & $42.35 \%$ & $49.15 \%$ & $52.18 \%$ \\
\hline 7 & $56.47 \%$ & $42.35 \%$ & $54.23 \%$ & $57.44 \%$ \\
\hline 8 & $60.46 \%$ & $42.35 \%$ & $58.77 \%$ & $62.08 \%$ \\
\hline 9 & $64.43 \%$ & $42.35 \%$ & $63.37 \%$ & $66.72 \%$ \\
\hline 10 & $70.02 \%$ & $42.36 \%$ & $69.13 \%$ & $72.29 \%$ \\
\hline 11 & $73.03 \%$ & $42.36 \%$ & $72.62 \%$ & $75.80 \%$ \\
\hline 12 & $75.33 \%$ & $72.59 \%$ & $75.14 \%$ & $78.33 \%$ \\
\hline 13 & $77.45 \%$ & $72.59 \%$ & $77.49 \%$ & $80.66 \%$ \\
\hline 14 & $79.63 \%$ & $72.59 \%$ & $80.00 \%$ & $83.06 \%$ \\
\hline 15 & $82.59 \%$ & $72.60 \%$ & $82.96 \%$ & $85.89 \%$ \\
\hline 16 & $84.09 \%$ & $72.60 \%$ & $84.62 \%$ & $87.38 \%$ \\
\hline 17 & $85.22 \%$ & $72.60 \%$ & $85.93 \%$ & $88.54 \%$ \\
\hline 18 & $86.35 \%$ & $86.49 \%$ & $87.23 \%$ & $89.67 \%$ \\
\hline 19 & $87.71 \%$ & $86.49 \%$ & $88.75 \%$ & $90.93 \%$ \\
\hline 20 & $89.84 \%$ & $86.49 \%$ & $90.80 \%$ & $92.64 \%$ \\
\hline 21 & $90.81 \%$ & $86.49 \%$ & $91.84 \%$ & $93.39 \%$ \\
\hline 22 & $91.47 \%$ & $86.49 \%$ & $92.56 \%$ & $93.97 \%$ \\
\hline 23 & $92.12 \%$ & $86.50 \%$ & $93.27 \%$ & $94.53 \%$ \\
\hline 24 & $92.93 \%$ & $86.50 \%$ & $94.11 \%$ & $95.19 \%$ \\
\hline 25 & $94.25 \%$ & $94.04 \%$ & $95.15 \%$ & $96.02 \%$ \\
\hline 26 & $94.83 \%$ & $94.04 \%$ & $95.66 \%$ & $96.41 \%$ \\
\hline 27 & $95.17 \%$ & $94.04 \%$ & $96.02 \%$ & $96.68 \%$ \\
\hline 28 & $95.52 \%$ & $94.04 \%$ & $96.38 \%$ & $96.96 \%$ \\
\hline 29 & $95.91 \%$ & $94.04 \%$ & $96.80 \%$ & $97.28 \%$ \\
\hline 30 & $96.54 \%$ & $94.05 \%$ & $97.35 \%$ & $97.71 \%$ \\
\hline 31 & $96.83 \%$ & $96.82 \%$ & $97.58 \%$ & $97.91 \%$ \\
\hline 32 & $97.02 \%$ & $96.82 \%$ & $97.76 \%$ & $98.06 \%$ \\
\hline 33 & $97.21 \%$ & $96.82 \%$ & $97.93 \%$ & $98.21 \%$ \\
\hline 34 & $97.42 \%$ & $96.82 \%$ & $98.14 \%$ & $98.39 \%$ \\
\hline 35 & $97.74 \%$ & $96.82 \%$ & $98.39 \%$ & $98.59 \%$ \\
\hline 36 & $97.89 \%$ & $96.82 \%$ & $98.51 \%$ & $98.71 \%$ \\
\hline 37 & $98.02 \%$ & $98.43 \%$ & $98.62 \%$ & $98.80 \%$ \\
\hline 38 & $98.14 \%$ & $98.43 \%$ & $98.73 \%$ & $98.89 \%$ \\
\hline 39 & $100.00 \%$ & $100.00 \%$ & $100.00 \%$ & $100.00 \%$ \\
\hline
\end{tabular}

Note: The sample comprises selected NYSE-listed common stocks included in the decimal pilot and their corresponding NYSE-listed control stocks over the decimal trial and all-decimal periods. The quoted bid-ask spreads are denominated in cents and the cumulative percentages (Cum\%) are computed from the $\mathrm{BBO}$ prices in the various exchanges. Only quoted spreads of up to $\$ 1$ are considered for this table. About $99.9 \%$ of all quoted BBO spreads are within $\$ 1$. 
the all-decimal period, we see that the spread dispersion at 12 cents or less declines, whereas it improves at 13 cents or more, both relative to the decimal trial period. The dispersion of the control stock spreads shows a marked improvement over the all-decimal period relative to the decimal trial period when they were trading in sixteenths. Thus, about $42 \%(52 \%)$ of the spreads in control stocks were at 6 cents or less over the decimal trial (all-decimal) period. Furthermore, $73 \%(78 \%)$ of the spreads in control stocks were at 12 cents or less over the decimal trial (all-decimal) period. Overall, it should be comforting to the supporters of decimal pricing that quote increments of 5 cents or less appear to be used actively by the market in a post-decimal world.

Tables 2 and 3 provide information on the quoted and the effective bid-ask spreads, in cents, on the NYSE-listed decimal stocks and the corresponding control stocks over all three periods considered in the study. Both the quoted and effective bid-ask spreads are calculated on the basis of the BBO quotes available at the time of trade. Thus, autoquotes, which simply bracket an existing BBO, are automatically eliminated from consideration. We compute spreads of stock portfolios formed on the basis of the average daily dollar volume of trade of stocks over the pre-decimal period. Stocks in portfolio 1 (5) constitute the smallest (largest) dollar volume stocks. The reported spreads in Table 2 (and in Panel A of Table 3) for each dollar volume portfolio are computed by weighting, for a given stock within a given day, by the time each spread is outstanding, and by weighting across stocks, by their daily average pre-decimal dollar trading volume.

Table 2 reveals that the decrease (over the decimal trial period) in quoted spreads for decimal stocks is significant across all five dollar volume portfolios, and it ranges from $26 \%$ to $36 \%$, whereas the dollar volume-weighted average decline over all portfolios is about $35 \%$. The corresponding decline in the control stock portfolios ranges from $2 \%$ to $16 \%$, with an overall average decline of about $16 \%$. Thus, decimalization appears to have significantly reduced quoted spreads as compared with control stocks.

To isolate the effect of decimalization on the quoted spreads of decimals stocks, the net difference in quoted spreads is calculated as the difference between the daily average quoted spread of each decimal stock and its paired control stock in a particular size rank. For each portfolio, the average of these differences over each period is then computed. The average of the differences, within each portfolio, is reported in Table 2. These reveal that decimalization itself may have resulted in quoted spreads' declining by an average of about 2.2 cents. The last column of Table 2 confirms that the difference in quoted spreads between decimal and control stocks is statistically insignificant over the all-decimal period.

Table 3, Panel A, reports the effective spreads of portfolios, once again classified by their average daily pre-decimal dollar volume. The reduction in effective spreads in decimal stocks ranges from 19\% (portfolio 5) to $30 \%$ (portfolio 1). The greatest reductions occur in the lower dollar volume portfolios, which, as Table 2 indicates, have relatively wider quoted spreads. The overall (dollar 


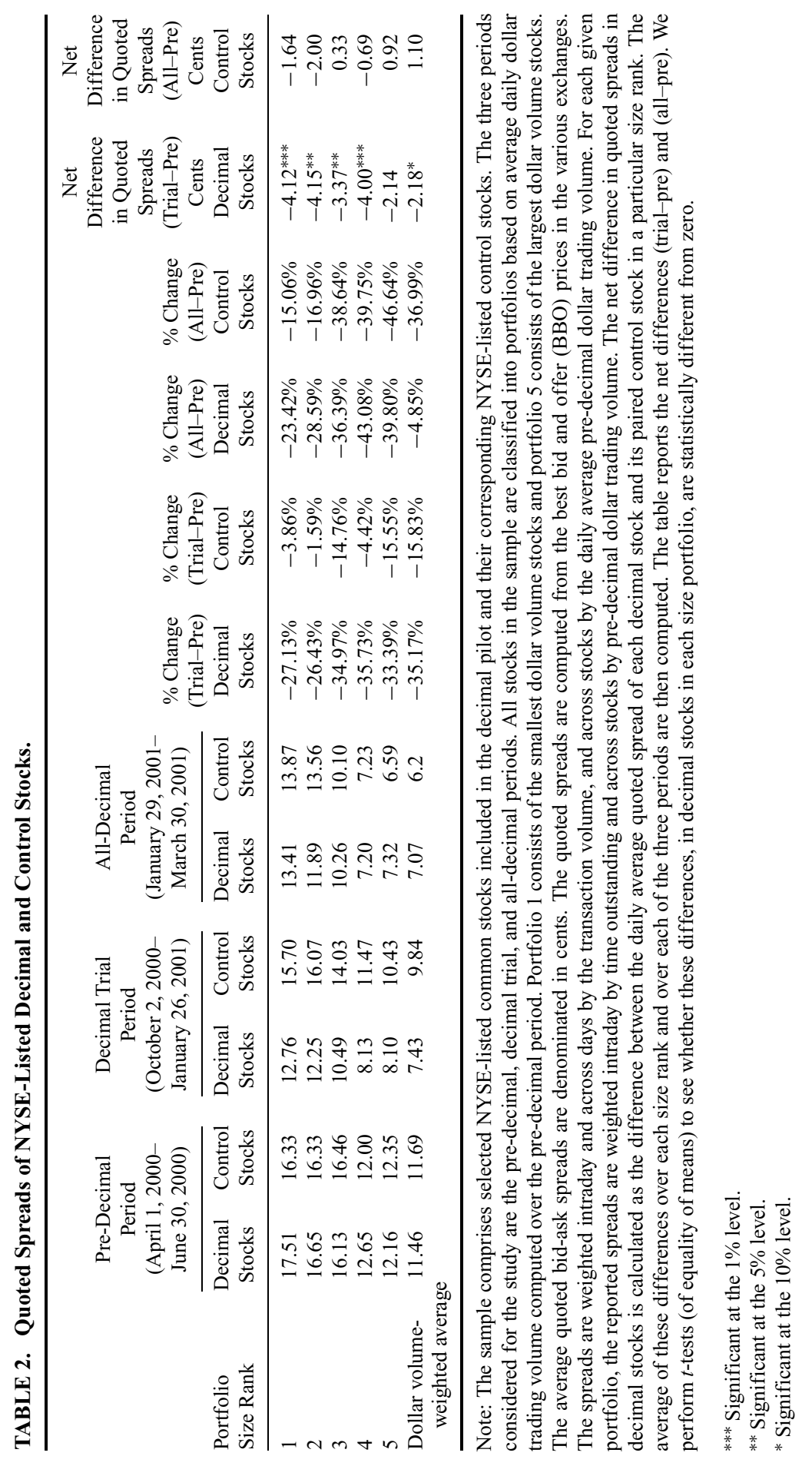




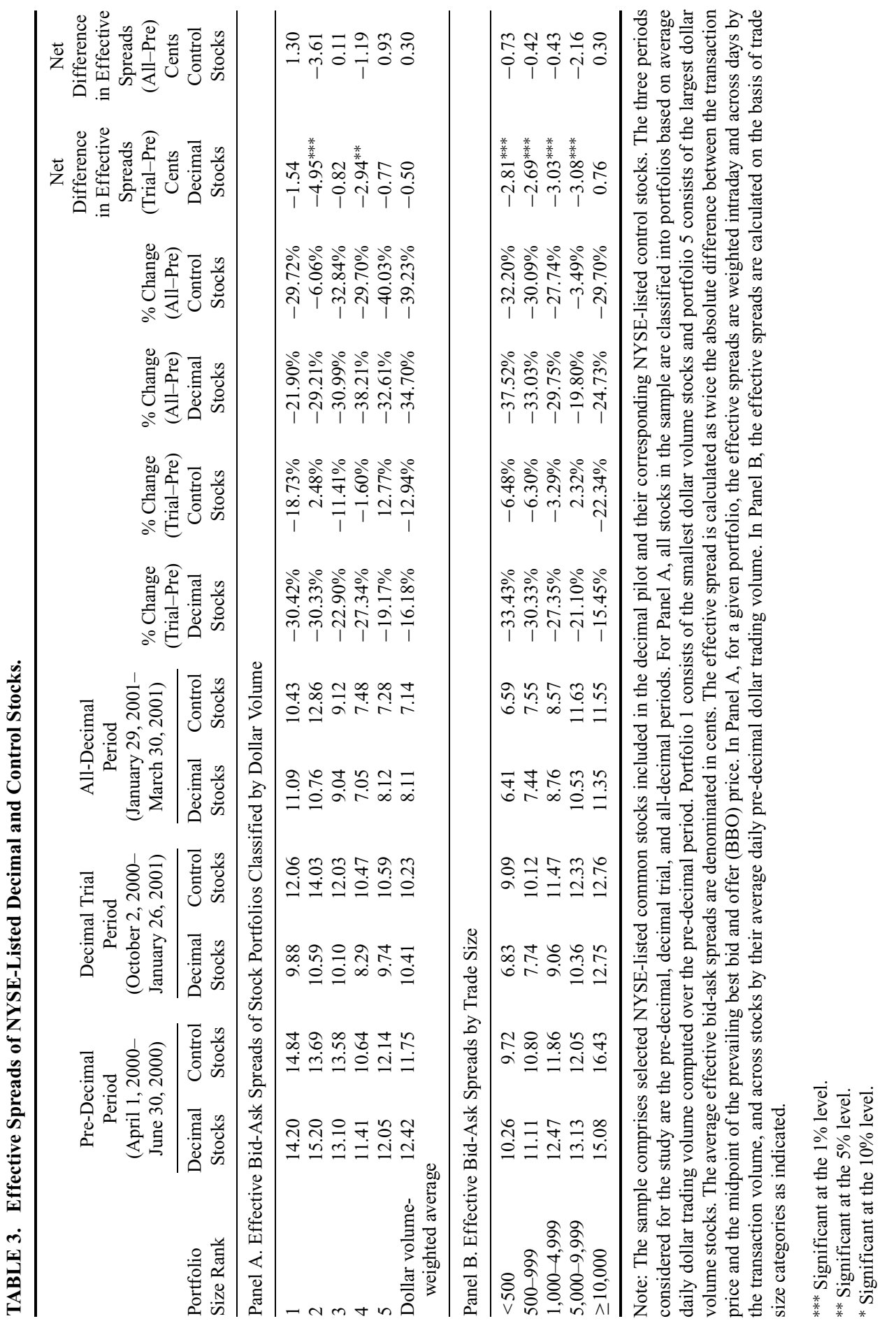


volume-weighted average) decrease is about $16 \%$. The control portfolios also show a decline in effective spreads in almost all portfolio size categories with an overall (dollar volume-weighted average) decrease of about 13\%. Furthermore, the trend of declining effective spreads continues over the all-decimal period, especially in the higher dollar volume portfolios. To isolate the effect of decimalization on the effective spreads of decimal stocks, the net difference variable is reported. Only portfolios 2 and 4 show a significant decline, whereas the rest are not significantly different from zero.

To get a sense of the relation between effective spreads and trade size, we further compute the effective spreads corresponding to trades in each of five trade size categories: trades less than 500 shares (small trades), trades between 500 and 999 shares (medium1 trades), trades between 1,000 and 4,999 shares (medium2 trades), trades between 5,000 and 9,999 shares (medium3 trades), and trades of 10,000 shares or greater (large trades). Table 3, Panel B, presents the average effective spreads in each trade size category, for decimal and control stocks, over the three periods of study.

We see that spreads in each trade size category are statistically similar between decimal and control stocks over the pre-decimal period, and there are significant declines in effective spreads in almost every trade size category in decimal stocks (relative to control stocks) over the decimal trial period. Specifically, the spread decreases monotonically from $33 \%$ in small trades to about $15 \%$ in large trades. In contrast, the decline is much more muted in control stocks over the same period. The net difference variable indicates that decimalization itself results in reduction of effective spreads ranging from 2.8 cents to 3.1 cents in all but the largest trade size category. For large trades, however, the net difference variable is not statistically significant at the $10 \%$ level, indicating no significant effective spread change ascribable to decimalization itself.

Following the path of effective spreads over the all-decimal period, we see a continuation in the downward trend in all trade size categories. This provides further support of improving liquidity in asset markets in the wake of decimalization.

In sum, decimalization appears to have significantly reduced both quoted and effective spreads in all but the largest size stocks, and this decline continues significantly beyond the start of the decimal pilot, indicating that the decline is not just a temporary phenomenon. Our conclusion holds even after accounting for effects other than decimalization and are consistent with those reported in Goldstein and Kavajecz (2000) and Jones and Lipson (2001) following the conversion to sixteenths. Decimalization appears to have also enabled relatively smaller size trades to obtain better prices and execute deeper inside the quoted spreads.

\section{Changes in Bid and Ask Depths}

A complete characterization of market liquidity must encompass both the bid-ask spreads and the corresponding bid and ask depths. When liquidity is defined 
along these two dimensions, it is likely that a reduction in liquidity could occur through a reduction in the bid or ask depth even though the bid-ask spread itself remains unchanged. Table 4 provides information on depths of the decimal and control stocks over the three periods studied, where depth is the average of the bid and ask depths in 100-share units.

We see that depths decreased significantly during the decimal trial period relative to pre-decimal period. The average decline in decimal stocks over the decimal trial period is about $69 \%$. The decline is the greatest (least) in the more active (less active) higher (lower) dollar volume stock portfolios. As the table indicates, the prevailing depths in the lowest dollar volume portfolio are the smallest to begin with. There is thus less room for improvement in the smallest stocks. In contrast, the control stock portfolios over the same period show an overall increase in depths (by about 14\%). Once again, the net difference variable isolates the effect of decimalization on the depths of decimal stocks following decimalization. We see that decimalization itself leads to a reduction in depth of about 9,742 shares. This trend over the decimal trial period continues into the all-decimal period, especially in the lower dollar volume stocks. Also, the difference in depth between the decimal stocks and control stocks is not statistically significant over the all-decimal period, which is as expected given that these are two matched groups of stocks trading in decimals.

In sum, after controlling for market trends (other than decimalization), there appears to be a significant decline in depth at the $\mathrm{BBO}$ and this decline continues long after the commencement of the decimal pilot, especially for less active stocks. Goldstein and Kavajecz (2000) report an average quote depth decline of about $48 \%$ following conversion to sixteenths. Although a decline in quoted and effective spreads indicates an increase in market liquidity, a simultaneous decrease in the corresponding depths (i.e., the corresponding order sizes for which these improved quotes are valid) implies a drop in liquidity. The overall effect of decimalization on market liquidity is therefore uncertain.

\section{Decimalization and Transaction-Related Variables}

\section{Trades and Trading Volume}

Decimalization could have differing effects on the size of the trade. To investigate the relative activity of large trades versus small trades around decimalization, we investigate the frequency of trades and trading volume in the five trade size categories described earlier. Table 5 reports results for both decimal and control stocks over the three periods of interest. Panel A presents the average daily trading volume results classified by trade size; Panel B provides the corresponding daily average trade frequency statistics. 


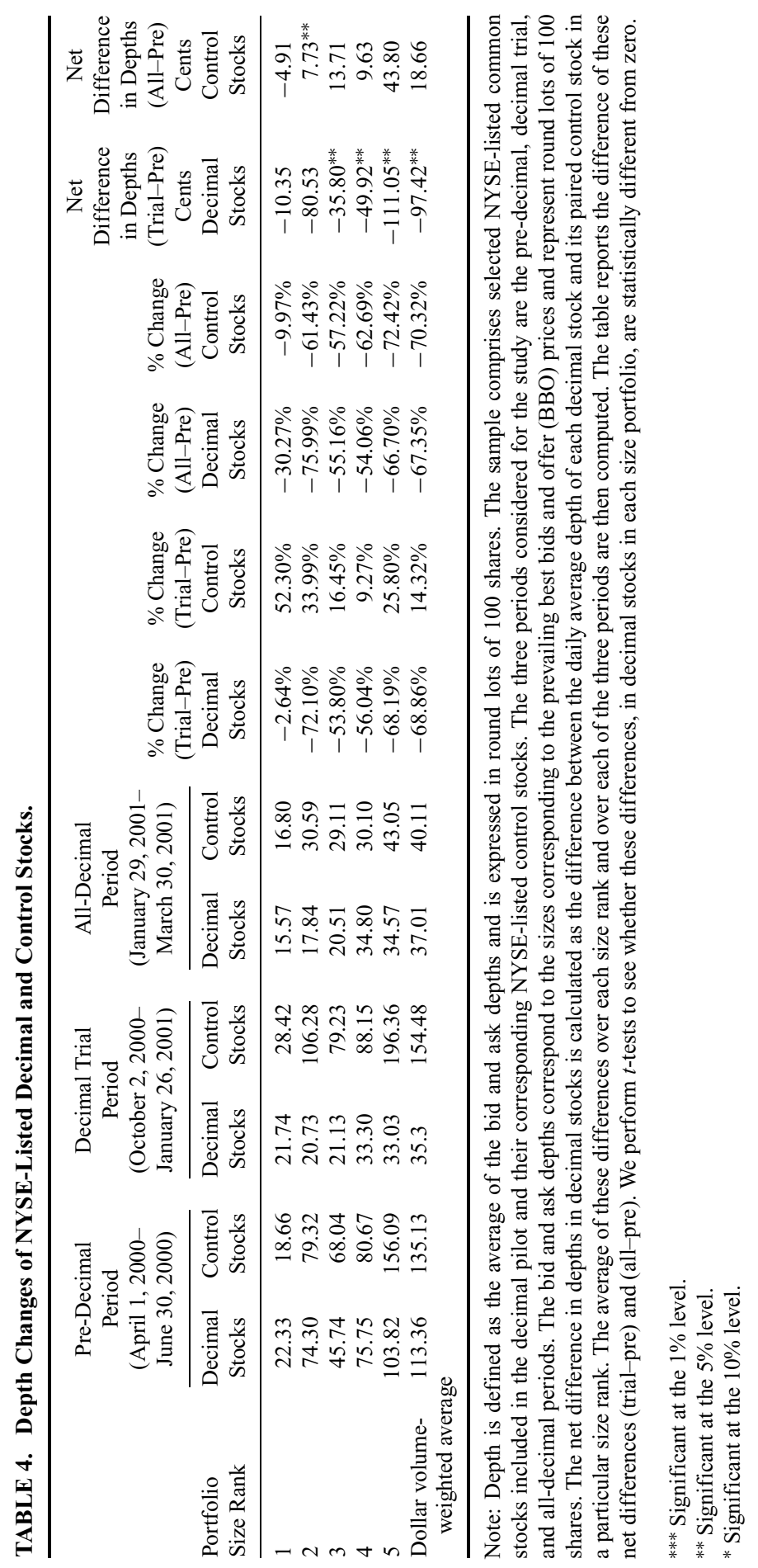




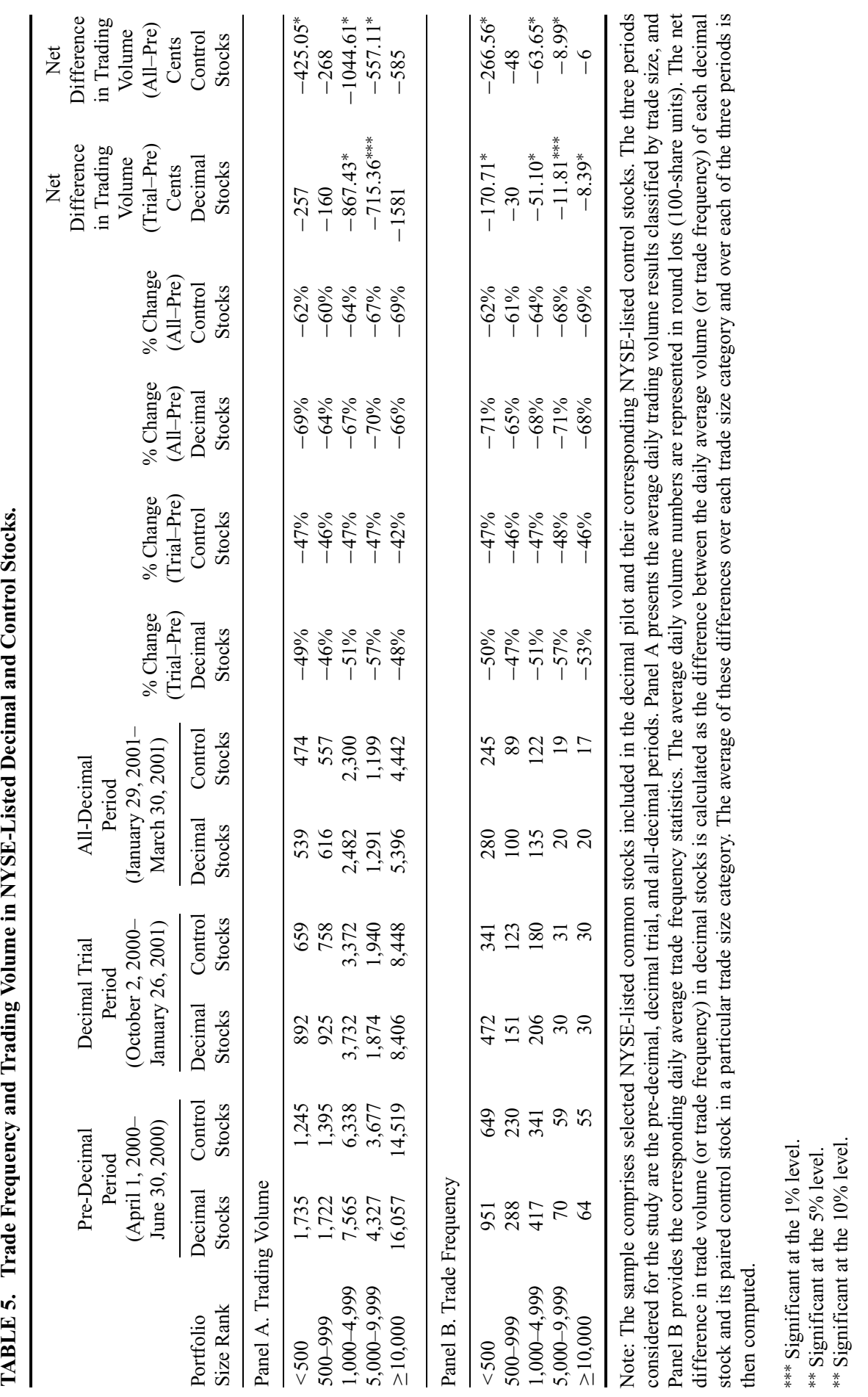


From Table 5 we see a decrease in average daily trades and trading volume in all trade size categories among decimal stocks in the decimal trial period. The control stocks display the same pattern and a similar magnitude of decline. On tracking the trade and trading volume pattern through the all-decimal period, we see a further decline in all trade size categories. To isolate the effects of decimalization, we report the net difference in trade volume (or trade frequency) in decimal stocks, calculated as the difference between the daily average volume (or trade frequency) of each decimal stock and its paired control stock in a particular trade size category. These numbers indicate that decimalization led to a significant decrease in trades and trading volume in medium trades. There is evidence that the frequency of large trades also declined following decimalization.

\section{Volatility}

If the risk of trading in decimal stocks has increased significantly (relative to the control stocks) following decimalization, we would expect a greater price effect (or higher volatility) for a trade of a given size in decimal stocks. We use the five portfolios of decimal and control stocks separately based on the average daily dollar trading volume over the pre-decimal period. We form a minute-by-minute return series for the stocks within these portfolios, where each return is weighted by its corresponding share volume. Portfolio returns are then formed by weighting each stock in a portfolio by its pre-decimal average daily dollar volume. Overnight returns are discarded. Volatility for portfolio returns is calculated daily and the average volatility across days is reported.

Table 6 reveals an increase in volatility across all decimal stock portfolios over the decimal trial period. This increase is small in the smallest dollar volume portfolio, becomes larger in the intermediate size portfolios, and declines for the largest portfolio. In contrast, the control stocks display a universal decline in volatility over the same period. The net difference variable attempts to isolate the effects of decimalization on decimal stocks. The results indicate that decimalization has led to a significant increase in volatility in all but the smallest portfolio.

Following the path of volatility in the two groups of stocks over the alldecimal period, we find a significant decline in volatility in both groups of stocks relative to the decimal trial period. This could indicate a reduction in decimal-related risk in the market as participants learn the new trading environment.

\section{Concluding Discussion}

Even though it is tempting to think of decimalization as just another decrease in minimum tick size, it is in reality much more than that. Decimalization makes it very cheap, $\$ 0.01$, for someone to step up in front of another liquidity supplier. Although this was true even up to the conversion to sixteenths, it may not 


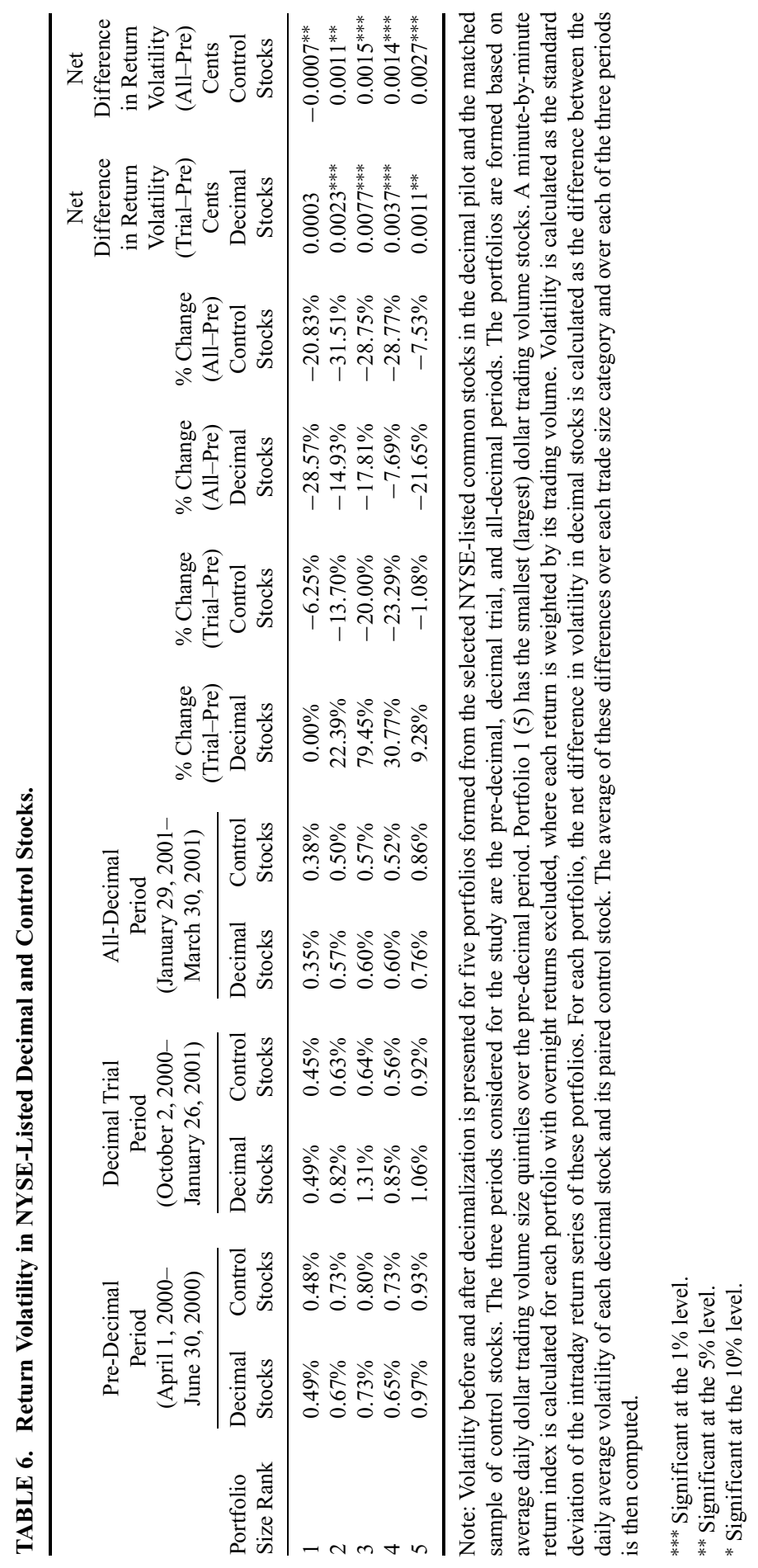


be true now, where it takes just 1 cent to step ahead of a standing limit order. If it is almost costless to step ahead, as one fund manager puts it, "mutual funds may stop using limit orders and simply pay fees to brokers to buy and sell at the prevailing market price. Most stocks already are traded that way on the nation's exchanges." Q3 With this backdrop-and using tick-by-tick transaction and quote data over three periods related to before, during, and after decimalization on the NYSE — we study the effect of decimalization on some of the common metrics of market liquidity, price and order competition, and the effect of regional versus the national exchanges. To isolate the effects of decimalization, we construct a sample of control stocks matched with each decimal stock in our sample.

We find mixed evidence related to market liquidity following decimalization. Specifically, both the quoted and effective bid-ask spreads as well as the quoted depth exhibit a significant decline. Thus, although better prices now exist to buy and sell, the quantities that can be purchased or sold at those improved prices are also fewer. Our finding of smaller spreads following decimalization bodes well for retail traders who trade in smaller sizes. We find that both trades and trading volume decline significantly in all trade size categories as well as in all stock size categories. Stock return volatilities show an increase in the short term but a decrease in the long term. This may imply a degree of comfort experienced by market participants as they learn to navigate their way around in the new environment.

Although we find some increased activity among the regional stock exchanges in terms of bid-ask quote adjustment frequencies, BBO times, and percentage of autoquotes, the evidence is not strong and there is no evidence to suggest that the market share of transactions executed by the regionals has increased as a result. The NYSE appears to be leading the way in terms of 1 cent to 5 cent changes in bids and offers among all exchanges as well as in (dollar) transactional volume.

One implication of our research is that retail investors are probably paying less to transact in a decimal world. These are investors whose trade sizes do not usually exceed the prevailing bid or ask sizes quoted at the improved (or tighter) prices. What it does for institutional investors' trading costs is unclear because these trades are typically large and require significant inroads into the limit order book or the presence of other suppliers of liquidity, typically in the upstairs market. Institutional trades are also distinct in that they require multiple trades, sometimes spanning days, to complete. In that regard, Chakravarty, Panchapagesan, and Wood (2001) examine institutional execution costs around decimalization in the NYSE using a large sample of institutional trades. They find no increase in trade execution costs in institutional trades after decimalization. Our results, combined with those of Chakravarty, Panchapagesan, and Wood, should be of some comfort to regulators who are under pressure to roll back decimalization and move to 5 cent ticks. ${ }^{7}$

${ }^{7}$ See "Decimal Move Brings Points of Contention from Traders," Wall Street Journal, February 12, 2001, p. C1, and "Deals \& Deal Makers: Grasso Says NYSE Must Stick to Penny as Trade Increment," Wall Street Journal, March 22, 2001, p. C18. 


\section{References}

Bacidore, J., R. Battalio, and R. Jennings, 2001a, Order submission strategies, liquidity supply, and trading in pennies on the New York Stock Exchange, NYSE working paper.

Bacidore, J., R. Battalio, and R. Jennings, 2001b, Changes in order characteristics, displayed liquidity, and execution quality on the New York Stock Exchange around the switch to decimal pricing, Working paper, Indiana University.

Bessembinder, H., 2001, Quote-based competition and trade execution costs in NYSE-listed stocks, Journal of Financial Economics.

Bessembinder, H., 2002, Trade execution costs and market quality after decimalization, Journal of Financial and Quantitative Analysis.

Blume, M. and M. Goldstein, 1997, Quotes, order flow, and price discovery, Journal of Finance 52, 221-44.

Chakravarty, S., V. Panchapagesan, and R. Wood, 2001, Institutional trading patterns and price impact around decimalization, Working paper, Purdue University.

Chordia, T., R. Roll, and A. Subrahmanyam, 2001, Market liquidity and trading activity, Journal of Finance $56,501-30$.

Chordia, T. and A. Subrahmanyam, 1995, Market making, the tick size, and payment-for-order flow: Theory and evidence, Journal of Business 68, 543-75.

Chung, K., B. Van Ness, and R. Van Ness, 1999, Limit orders and the bid-ask spread, Journal of Financial Economics 53, 255-87.

Chung, K., B. Van Ness, and R. Van Ness, 2002, Trading costs and quote clustering on the NYSE and Nasdaq after decimalization, Working paper, SUNY at Buffalo.

Goldstein, M. and K. Kavajecz, 2000, Eights, sixteenths and market depth: Changes in tick size and liquidity provision on the NYSE, Journal of Financial Economics 56, 125-49.

Harris, L., 1994, Minimum price variations, discrete bid ask spreads, and quotation sizes, Review of Financial Studies 7, 149-78.

Hasbrouck, J., 1995, One security, many markets: Determining the contributions to price discovery, Journal of Finance 50, 1175-99.

Jones, C. and M. Lipson, 2001, Sixteenths: Direct evidence on institutional execution costs, Journal of Financial Economics 59, 253-78.

McInish, T. and R. Wood, 1986, Adjusting for beta bias: An assessment of alternative techniques: A note, Journal of Finance 46, 277-86. 